\title{
Molecule of Equivalent Soluble Fluorochrome
}

National Cancer Institute

\section{Source}

National Cancer Institute. Molecule of Equivalent Soluble Fluorochrome. NCI Thesaurus. Code C96691.

A unit of measure of the fluorescence intensity of a fluorochrome-labeled sample, which is equivalent to the fluorescence intensity of a solution containing an equivalent number of molecules of free fluorochrome in solution, under identical experimental conditions. 